\title{
Immunopathology of mycobacterial diseases
}

\author{
Stefan H. E. Kaufmann ${ }^{1}$
}

Received: 2 November 2015 / Accepted: 2 November 2015 /Published online: 2 February 2016

(C) Springer-Verlag Berlin Heidelberg 2016

The granuloma is the hallmark of tuberculosis (TB), at least from the standpoint of pathology. Accordingly, this special issue of Seminars in Immunopathology focuses on a better understanding of immunopathology of TB, with emphasis on the "tuberculoma" as the lesion had once been termed. In fact, the term tuberculoma was introduced before the designations of TB and granuloma.

Intrigued by the pathologic similarities of scrofulosis and phthisis, Franciscus Deleböe Sylvius (1614-1672) foresaw similarities between the swollen lymph nodes in scrofulosis and the pulmonary lesions in phthisis, and in fact, concluded "I hold that not infrequently phthisis has its origin from these tubercules" [1]. In a similar line, Jo Baptist Morgagni (16821771) considered similar etiology of phthisis and scrofulosis very likely [2]. It was Franciscus Deleböe Sylvius who coined the term "tubercle." Rudolf Virchow (1821-1902), in his book Cellularpathologie [3], introduced the term "granuloma" for lesions characterized by granulation and clear separation from healthy tissue. In his treatise, Die krankhaften Geschwülste, Virchow considered the word granuloma appropriate to describe such lesions [4-6]. In his view, the tuberculoma therefore was just one form of granuloma, which over the years replaced the specific term tuberculoma. However, it persists in describing the disease TB. This is due to

This is the introduction to Immunopathology of Mycobacterial Diseases Dr. Stefan H.E. Kaufmann

Stefan H. E. Kaufmann

kaufmann@mpiib-berlin.mpg.de

1 Department of Immunology, Max Planck Institute for Infection Biology, Charitéplatz 1, D-10117 Berlin, Germany
Lukas Schönlein (1793-1864), who rightly considered the tubercle as hallmark, and hence, appropriate root word for the clinical description of this disease [7].

Today, we know that granulomas are primarily composed of cells of the hematopoietic lineage, notably, mononuclear phagocytes and lymphocytes. A more careful analysis reveals dendritic cells and neutrophils, and both $\mathrm{T}$ and $\mathrm{B}$ lymphocytes. Current dogma holds that $\mathrm{T}$ lymphocytes and mononuclear phagocytes are the central collaboration partners in the lesion. Granulomas are induced whenever material cannot be degraded and eliminated in due time, be it infectious as in TB or noninfectious, as for example, in sarcoidosis. The tuberculoma is the site of both protection and pathology since on the one hand it constrains tubercle bacilli and on the other hand it affects tissue function. Obviously, this has to be seen in context: the solid and the fibrotic granulomas primarily protect, whereas the necrotic and even more so the liquefied lesions are primarily detrimental.

Since the elucidation of the contagious etiology of TB by Robert Koch (1843-1910) in 1882, the granuloma has been intensively studied from a microbiologic and immunologic perspective. In his original publication on the etiology of TB, Koch described the identification of the pathogen within granulomas and stated "all these factors together allow me to state that the bacilli present in the tuberculous lesions do not only accompany tuberculosis but rather cause it" [8]. Elie Metchnikoff(1845-1916), the famous discoverer of phagocytosis, identified mononuclear phagocytes as major effectors but under certain conditions also as habitat of Mycobacterium tuberculosis. In the following are a few highlights from his paper: "the real phagocytes (in particular macrophages) serve as critical defenders of the host against tubercle bacilli; ... without doubt the phagocytes are capable of engulfing live and virulent tubercle bacilli actively; ....and the giant cells are capable of killing these parasites; ... of course it is possible 
that tubercle bacilli can evade the deadly activity of giant cells" [9].

The elucidation of the contagious nature of TB by Robert Koch in 1882 was not only the turning point for TB research and for the development of diagnostics, therapeutics, and vaccines for this disease. It was also the foundation of the concept that infectious diseases are a distinct group of pathologies caused by exogenous invaders. This is best reflected in the Koch-Henle postulates defining contagious disease based on three observations [8].

1. Existence of a living microorganism in all affected tissues;

2. Isolation of the infectious agent from these tissues in clonal form;

3. Elicitation of disease of similar type in an experimental animal by transfer of the infectious agent.

Robert Koch made these statements while working in Berlin, where Rudolf Virchow propagated cellular pathology, meaning that diseases exclusively arise inside the cell. Exogenous invaders were not considered in this concept. That is, the tuberculoma as a structured aggregation of specific host cells was seen as testament for the endogenous origin of TB.

Identification of M. tuberculosis as agent of TB has led to the development of diagnostics and drugs targeting the pathogen. These weapons unfortunately have become blunt, and today, efforts are increasingly focused on the host response. These include diagnostics based on global host-gene expression profiles, so-called biomarkers [10], and host-directed therapies reverting mycobacterial subversion and misuse of host effector mechanisms by small molecules or by monoclonal antibodies. Not the least, this is the outcome of intensified research on immunopathology of TB ranging from basic research to applied investigations.

This special volume builds on the shoulders of these giants and provides a state-of-the-art overview of the major immunopathologic mechanisms underlying TB. It goes without saying that the tuberculoma comprises a major part of this volume.

Qualls and Murray view the TB granuloma from a metabolic standpoint [11]. Over the past few years, the role of small molecules in immunoregulation has gained increasing interest. In TB, small molecules relevant to disease control in the granuloma include arginine, tryptophan, as well as reactive oxygen and nitrogen intermediates (ROI and RNI, respectively). Arginase 1 is a checkpoint enzyme since by metabolizing L-arginine it modulates RNI production, T cell proliferation, and tryptophan degradation. Arginase 1, in turn, is induced by lactate. Both $M$. tuberculosis and immunity in TB are oxygendependent, and therefore, oxygen is essential for ROI and RNI secretion and also needed by the pathogen, which can however, survive in areas of hypoxia. Investigations into the local metabolic network in tuberculomas will not only reveal novel insights into the crosstalk between host and pathogen at the site of action but also provide important information about novel therapeutic concepts. First, the activity of several conventional TB drugs is highly dependent on oxygen pressure in the granuloma, and second, manipulation of the metabolic balance within granulomas can be harnessed for host-directed interventional strategies.

Although the tuberculous granuloma can only fully develop under the guidance of the acquired $\mathrm{T}$ cell response, innate mechanisms initiate and promote this development at the early stage of infection. With reemerging interest in the innate immune system as an instructor of acquired immunity, relevant mechanisms have been elucidated. These include the pro- and anti-inflammatory cytokines, chemokines, lipid mediators, and type I interferons, which all play critical roles in granuloma formation. Further development of granulomas towards fibrosis or liquefaction again is stringently orchestrated by soluble mediators including, in addition to cytokines and chemokines, enzymes, notably members of the matrix metalloprotease family and cognate cell-cell interactions, which can be costimulatory or coinhibitory. As discussed by Dorhoi and Kaufmann, these novel insights can offer novel directions for host-directed therapeutics for TB, some of which have already entered clinical application for other diseases, and hence, allow repurposing for TB therapy [12].

A major theme of this volume is the role of the granuloma in protection and pathogenesis in TB. Even though there is still a long way to go until this complex tissue reaction is fully deciphered, first glimmers of light offer hope for directed drug targeting of granulomas. Kiran, Podell, Chambers, and Basaraba describe potential checkpoints for such approaches. It is likely that these therapies serve as adjunct to conventional rather than stand-alone therapeutics [13]. Potential approaches include mechanisms favoring formation of solid granulomas over necrosis and caseation, mechanisms that improve effectiveness of current chemotherapy, mechanisms that prevent spread of M. tuberculosis, and mechanisms improving tissue modeling and healing.

The human immunodeficiency virus (HIV) is not only the driving force for TB but the latter is also the number one cause of death in HIV-infected individuals. As a further complication, immune reconstitution inflammatory syndrome (IRIS) can occur after initiation of antiretroviral therapy (ART). Although incompletely understood, this pathology is mediated by a hyperactivation of the immune response after reduction of viral load. It comprises various cytokines and lymphocyte populations. Lai, Meintjes, and Wilkinson review our current knowledge of this pathologic reaction, which can provide the basis for urgently needed immune intervention measures to overcome adverse events of this essential treatment option of the one million HIV coinfected people developing TB every year [14].

Given the critical role of immunity in TB, host genetics must play a major role. Indeed, it is ironic that both Koch 
and Virchow were right. Without doubt, TB is an infectious disease, but its underlying pathology is strongly influenced by host cell genetics. This is best illustrated by the fact that only a small proportion of those who become infected develop active disease, while the majority remains healthy with latent TB infection. Yet, inheritance of TB susceptibility is not as frequently found as one would assume. This is partly due to the fact that on the one hand diagnosis of inherited diseases is more sophisticated and, therefore, performed mostly in industrialized countries, where on the other hand risk of exposure to M. tuberculosis is relatively low. Therefore, more information is available on inheritance correlation with non-tuberculous mycobacterial infections and BCGosis [generalized bacille Calmette-Guérin (BCG) disease in areas where BCG immunization is performed]. Wu and Holland [15] discuss the role of simple and complex genetic disorders in susceptibility to mycobacterial diseases. While monogenic defects are most frequently seen for disseminated mycobacterial disease, lung disease caused by non-tuberculous mycobacteria is highly complex including deficiencies that affect pulmonary tissue functions. Studies focused on BCG and non-tuberculous mycobacteria provide extremely important information about relevant immune mechanisms. Yet, the more specific mechanisms, which exclusively control pulmonary TB as the most prevalent form of disease, remain enigmatic.

The role of the causative agent of TB, M. tuberculosis, is the focus of the chapter by Martin, Carey, and Fortune [16]. They describe how mycobacteria respond to the unique microenvironment they face at different sites within the granuloma as well as mycobacterial impact on the fate of the granuloma. It is becoming increasingly clear that the type of granuloma that evolves is not only a matter of the host response but also of the pathogen, i.e., the crosstalk between both. Much needs to be understood about these mechanisms and how they drive caseation in one lesion and sustain the solid structure in another lesion, in one and the same patient. Current evidence suggests that these polarizations are not stochastic but controlled, although the underlying mechanisms remain elusive.

Experimental TB of mice has been instrumental for our understanding of basic immune mechanisms in TB. Yet, the granuloma that develops in conventional mice does not fully reflect the composition and variability of human granulomas. Most importantly, the pathologic mechanisms in murine and human lesions vary significantly. The murine lesion is considered a simpler version that develops over the course of infection but does not progress into polar forms as seen in human TB, where a spectrum of solid, necrotic, and caseous granulomas is seen. However, recent advances allow harnessing the genetic heterogeneity of different mouse strains generated by breeding or by genetic manipulation of mice. Kramnik and Beamer describe such models, which now make it possible to combine our increasing knowledge about the immune response in mice with models that more realistically mirror the pathologies of human lesions in TB [17]. There is no doubt that such mouse models will not only promote better mechanistic understanding of granuloma formation and maintenance but also form the basis for a pipeline of novel host-directed interventions.

The term mycobacterium indicates phenotypic similarities with fungi. After all, the term mycology describes the biology of fungi and medical mycology focuses on fungal diseases. Although Mycobacteriacae are clearly grouped as genus of Actinobacteriacae, lessons can be learned from fungal infections. Zelante, Pieraccini, Scaringi, Aversa and Romani describe the role of major metabolites, notably, tryptophan metabolites generated through host indoleamine 2,3-dioxygenase 1 (IDO1) and its crosstalk with the aryl hydrocarbon receptor (AhR) in fungal infections [18]. Many fungi are commensals of the human host which have to find their niche in the complex world of the microbiota. Although the fungal biome is just being elucidated, it is obvious that it will reveal novel insights into the tripartisan crosstalk between: bacterial and fungal commensals, bacterial/fungal pathogens, and the host immune system. Protective immunity to many fungal pathogens depends on a balanced type $1 \mathrm{~T}$ helper (Th1)-Th17 cell response with a strong participation of the latter. In TB, Th1 and Th17 responses are also activated and need to be well balanced but with a preponderance of the Th1 response. This rather simple immunologic network of CD4 T cells already illustrates the complexity that can be easily overlooked in systems that only study a single pathogen, considering neither the microbiome/mycobiome nor coinfections.

Granulomas are widespread and occur whenever infectious or noninfectious material cannot be eliminated in due time. Although the tuberculous granuloma is often considered the paragon of all granuomatous lesions, it is worth learning from other examples. Here, Kaye and Beattie describe lesions in leishmaniasis [19]. Although these lesions are simpler in structural organization than tuberculomas, they provide significant insights into the immune regulation of granuloma formation and protection. This may form the basis for host-directed therapy of leishmaniasis based on checkpoint regulation as has been done so successfully in certain cancer treatments.

Meijer describes a more recent innovative model to study pathology of TB notably in the dynamic context of granuloma formation and maintenance [20]. This is the zebrafish model, which has already proven its high value. The advantages are obvious. Zebrafish are naturally infected by $M$. marinum, a close relative of $M$. tuberculosis; embryos and larvae of zebrafish are translucent, and, therefore allow noninvasive evaluation of the developing granuloma. Genetic manipulation of zebrafish is far easier than in the mammalian system. Taken together, the zebrafish model provides new insights into the pathology of TB and can be harnessed as a highthroughput system for the evaluation of novel drugs, notably for host-directed therapy. 
This treatise summarizes recent highlights in the area of immunopathology of TB. Although basic mechanisms are the major topic, this information will form the basis for novel intervention measures beyond conventional developments. Such measures are urgently needed to fulfill the goal of the StopTB Partnership within the World Health Organization to reduce morbidity (9.6 million new cases in 2014) and mortality (1.5 million deaths in 2014) of TB, by 90 and $95 \%$, respectively, by the end of 2035 [21].

\section{References}

1. Deleboe FS (1679) Opera Medica. Danielum Elsevirium et Abrahamum Wolfgang, Amsterdam

2. Morgagni G (1765) De sedibus, et causis morborum per anatomen indagatis libri quinque, 2nd edn. Sumptibus Remondinianis, Patavii

3. Virchow R (1871) Die Cellularpathologie in ihrer Begründung auf physiologische und pathologische Gewebelehre, 4th edn. August Hirschwald, Berlin

4. Virchow R (1863) Vorlesungen über Pathologie: Die Krankhaften Geschwülste, vol 1. August Hirschwald, Berlin

5. Virchow R (1864) Vorlesungen über Pathologie: Die Krankhaften Geschwülste, vol 2. August Hirschwald, Berlin

6. Virchow R (1865) Vorlesungen über Pathologie: Die Krankhaften Geschwülste, vol 3a. August Hirschwald, Berlin

7. Schönlein JL (1841) Allgemeine und specielle Pathologie und Therapie. Literatur-Comtoir, St. Gallen

8. Koch R (1882) Die Aetiologie der Tuberculose (Nach einem in der physiologischen Gesellschaft zu Berlin am 24.März gehaltenem Vortrage). Berl Klin Wochenschr 19:221-230

9. Metschnikoff E (1888) Ueber die phagocytäre Rolle der Tuberkelriesenzellen. Virchows Arch Pathol Anat Physiol Klin Med 113:63-94
10. Weiner J, Kaufmann SH (2014) Recent advances towards tuberculosis control: vaccines and biomarkers. J Intern Med 275(5):467480

11. Qualls JE, Murray PJ (2015) Immunometabolism within the tuberculosis granuloma: amino acids, hypoxia, and cellular respiration. Semin Immunopathol. doi:10.1007/s00281-015-0534-0

12. Dorhoi A, Kaufmann SH (2015) Pathology and immune reactivity: understanding multidimensionality in pulmonary tuberculosis. Semin Immunopathol. doi:10.1007/s00281-015-0531-3

13. Kiran D, Podell BK, Chambers MA, Basaraba RJ (2015) Hostdirected therapy targeting the Mycobacterium tuberculosis granuloma: a review. Semin Immunopathol. doi:10.1007/s00281-0150537-x

14. Lai RP, Meintjes G, Wilkinson RJ (2015) HIV-1 tuberculosis-associated immune reconstitution inflammatory syndrome. Semin Immunopathol. doi:10.1007/s00281-015-0532-2

15. Wu UI, Holland SM (2016) A genetic perspective on granulomatous diseases with an emphasis on mycobacterial infections. Semin Immunopathol. doi:10.1007/s00281-015-0552-y

16. Martin CJ, Carey AF, Fortune SM (2015) A bug's life in the granuloma. Semin Immunopathol. doi:10.1007/s00281-015-0533-1

17. Kramnik I, Beamer G (2015) Mouse models of human TB pathology: roles in the analysis and the development of hostdirected therapies. Semin Immunopathol. doi:10.1007/s00281015-0538-9

18. Zelante T, Pieraccini G, Scaringi L, Aversa F, Romani L (2015) Learning from other diseases: protection and pathology in chronic fungal infections. Semin Immunopathol. doi:10.1007/s00281-0150523-3

19. Kaye PM, Beattie L (2015) Lessons from other diseases: granulomatous inflammation in leishmaniasis. Semin Immunopathol. doi:10.1007/s00281-015-0548-7

20. Meijer AH (2015) Protection and pathology in TB: learning from the zebrafish model. Semin Immunopathol. doi:10.1007/s00281015-0522-4

21. WHO (2015) Global Tuberculosis Report 2015. WHO Press, Geneva 